\title{
SHEA neonatal intensive care unit (NICU) white paper series: Practical approaches to Staphylococcus aureus disease prevention
}

\author{
Ibukunoluwa C. Akinboyo MD¹, Kenneth M. Zangwill MD2, Wendy M. Berg BSN, RN, CIC ${ }^{3}$, Joseph B. Cantey MD ${ }^{4}$, \\ Beth Huizinga MSN RNC-NIC ${ }^{5}$ and Aaron M. Milstone MD, MHS 6 \\ ${ }^{1}$ Division of Pediatric Infectious Disease, Department of Pediatrics, Duke University School of Medicine, Durham, North Carolina, ${ }^{2}$ Division of Pediatric Infectious \\ Diseases, Department of Pediatrics, Harbor-UCLA Medical Center, Torrance, California, ${ }^{3}$ Department of Infection Prevention and Control, Children's Hospitals and \\ Clinics of Minnesota, St. Paul, Minnesota, ${ }^{4}$ Divisions of Pediatric Infectious Diseases and Neonatology, Department of Pediatrics, University of Texas Health \\ Science Center at San Antonio, San Antonio, Texas, ${ }^{5}$ Women and Infant Services, Helen DeVos Children's Hospital Neonatal Services, Grand Rapids, Michigan and \\ ${ }^{6}$ Division of Pediatric Infectious Diseases, Department of Pediatrics, Johns Hopkins University School of Medicine, Baltimore, Maryland
}

\section{Introduction}

This white paper provides clinicians with practical guidance on the implementation of Staphylococcus aureus infection prevention measures for neonatal intensive care unit (NICU) patients. Except where otherwise specified, "S. aureus" refers to both methicillin-resistant $S$. aureus (MRSA) and methicillin-susceptible S. aureus (MSSA). The phrase "parents or family members" refers to all visitors that have physical contact with infants in the NICU.

$S$. aureus is a clinically important pathogen among NICU infants. Neonates may acquire $S$. aureus as part of their normal developing microbiota; yet, colonization predisposes to invasive infection. ${ }^{1,2}$ Estimated incidence of $S$. aureus infection is up to 45 per 10,000 hospitalized infants. ${ }^{3-5}$ In a typical NICU, exposure to the NICU environment, including indirect transmission from other critically ill infants, healthcare personnel, parents and/or family members may exacerbate the burden of $S$. aureus colonization and infection. ${ }^{6,7}$ Despite decreasing device utilization rates within the NICU, $S$. aureus remains a frequent cause of healthcare-associated and device-associated infections. ${ }^{4}$ S. aureus is also a commonly identified pathogen among hospitalized neonates during infection clusters or outbreaks. ${ }^{8,9}$ Most available literature report on $S$. aureus prevention in the context of outbreak control; few data exist on this topic in endemic NICU settings.

\section{Intended use}

The Society for Healthcare Epidemiology of America (SHEA) intends for this document to serve as a companion to the Centers for Disease Control and Prevention (CDC) Healthcare Infection Control Practices Advisory Committee (HICPAC) Recommendations for Prevention and Control of Infections in Neonatal Intensive Care Unit Patients and to provide practical, expert opinion and/or evidence-based answers to frequently asked questions on $S$. aureus detection and prevention in the NICU. The published literature does not contain sufficient high-quality studies to meet Grading of Recommendations

Author for correspondence: Ibukunoluwa C. Akinboyo, E-mail: ica5@duke.edu Cite this article: Akinboyo IC, et al. (2020). SHEA neonatal intensive care unit (NICU) white paper series: Practical approaches to Staphylococcus aureus disease prevention. Infection Control \& Hospital Epidemiology, 41: 1251-1257, https://doi.org/10.1017/ ice. 2020.51
Assessment, Development and Evaluation (GRADE) standards and therefore were not included in the HICPAC guideline. ${ }^{10}$

No guideline, expert guidance, or white paper can anticipate all situations. This document is meant to serve as an adjunct to individual judgment by qualified professionals. In general, these recommendations apply to non-outbreak settings. Healthcare personnel may implement additional measures during an outbreak or other special clinical scenarios. Further details and references are included in the HICPAC Recommendations for Prevention and Control of Infections in Neonatal Intensive Care Unit Patients: Staphylococcus aureus.

\section{Methods}

This document was developed by a collaborative panel of pediatric and pathogen-specific experts, members of the HICPAC guidelinewriting panel, and the SHEA Pediatric Leadership Council, to identify practical questions anticipated from practitioners and infection prevention professionals.

Unlike the SHEA expert guidance format, this document is not based on a systematic literature search; instead, for the selected topic areas, the authors provide practical approaches in questionand-answer format. Answers are based on consensus expert opinion within the context of the literature search conducted for the HICPAC document and supplemented by other published information retrieved by the authors.

This document is part of a SHEA white paper series that includes the "SHEA neonatal intensive care unit (NICU) white paper series: Practical approaches to Clostridioides difficile prevention," published in August 2018. ${ }^{11}$ SHEA convened a group of experts in pediatrics and neonatology, called the NICU Advisory Panel (see the Acknowledgments section), to oversee the full white paper series. The NICU Advisory Panel members serve as representatives for the following organizations: SHEA, the American Hospital Association (AHA), the American Academy of Pediatrics (AAP), the Association for Professionals in Infection Control and Epidemiology (APIC), the Infectious Diseases Society of America (IDSA), The Joint Commission, the National Association of Neonatal Nurses (NANN), the Pediatric Infectious Diseases Society (PIDS), and the Vermont Oxford Network (VON). This document was reviewed by the NICU Advisory Panel, the SHEA Guidelines Committee, and the 
SHEA Publications Committee. It was endorsed by the Society for Healthcare Epidemiology (SHEA), the Association for Professionals in Infection Control and Epidemiology (APIC), the Infectious Diseases Society of America (IDSA), The Joint Commission, the National Association of Neonatal Nurses (NANN), the Pediatric Infectious Diseases Society (PIDS), and the Vermont Oxford Network (VON).

\section{Authors}

The authors include current and past members of the SHEA Guidelines Committee and the SHEA Pediatric Leadership Council. Ms. Berg served as the representative from APIC. All authors served as volunteers. The authors are directly involved or provide an advisory role, at their respective institutions, in the development of policies pertaining to pediatric and/or neonatal infection prevention in the NICU.

The NICU Advisory Panel (see the Acknowledgments section), a collaborative group of pediatric and pathogen-specific experts convened by SHEA, provided oversight and review of the draft document. The NICU Advisory Panel includes representatives from SHEA, AHA, AAP, APIC, IDSA, The Joint Commission, NANN, PIDS, and VON.

\section{Practical approaches: Questions and Answers}

Question 1: Should family members and visitors wear personal protective equipment (PPE) when visiting an infant on contact precautions for MRSA?

\section{Answer 1:}

1. Family members and visitors should perform proper hand hygiene upon entering and exiting the NICU, and before and after each contact with the infant(s).

2. Healthcare personnel may choose not to require family members and visitors to use PPE for infants with MRSA colonization.

Some NICUs require parents and visitors to wear PPE (ie, donning of gowns and gloves) if their infant has been placed on contact precautions (eg, for MRSA colonization). Although some studies have shown that prevention bundles, including universal gown and gloving of healthcare personnel, can reduce the horizontal transmission of organisms, no compelling evidence exists to support routine PPE use by family members and visitors in the NICU. ${ }^{12}$ If family members and visitors comply with proper hand hygiene practices, transmission of MRSA from infants to family members and visitors should not result in horizontal transmission to other infants in the NICU. The potential benefit of routine gown and glove use by family members and visitors to prevent MRSA transmission does not outweigh the impact on care and bonding with the infant. No systematic studies have evaluated the use of PPE by family members and visitors within the NICU to reduce MRSA transmission. Furthermore, increasing evidence shows that skin-to-skin contact (kangaroo care) can support family member attachment, breastfeeding, and possibly long-term growth and development. Isolation gowns limit skin-to-skin care and have been described by family members as a barrier to attachment and breastfeeding.

Family members and visitors should be encouraged to perform hand hygiene when entering and leaving the NICU and immediately before and after visiting their infant(s). If family members and visitors have multiple infants being cared for in the NICU, then healthcare personnel should inform them about parents' possible role in the spread of MRSA between siblings. Healthcare personnel should monitor the parents to ensure that they comply with hand hygiene, and it may be prudent for them to visit the infants on contact precautions last (refer to discussion below about care of multiple gestations and discordant MRSA results). Family members and visitors should also be reminded to avoid contact with other families or other infants, including their surrounding bed space or medical equipment. This may be more challenging in units designed with open bays than in units with private rooms.

Question 2: If a parent is known to have MRSA infection or colonization, should healthcare personnel place infants on contact precautions, encourage skin-to-skin bonding, and/or institute breastfeeding restrictions?

\section{Answer 2:}

1. Contact precautions are not necessary for infants of MRSAcolonized or infected parents.

2. Healthcare personnel should emphasize hand hygiene to prevent transfer of organisms and consistently model the practice to parents and family members.

3. Healthcare personnel should encourage and promote skin-toskin care and breastfeeding while taking into consideration the location of the parent's MRSA lesion, ability to contain drainage, and likelihood of infant's contact with the lesion.

4. Parents known to be colonized with MRSA do not need to wear gowns or gloves while caring for their infant.

5. As long as drainage is contained, the site of infection is covered and strict hand hygiene practices are followed, healthcare personnel should not require parents known to have active MRSA infection(s) to wear gowns or gloves while caring for their infant(s).

6. Units should strongly consider routine screening of infant(s) (eg, weekly) whose parent has an active MRSA infection.

Managing the care of infants with an MRSA-colonized or -infected parent varies. Such infants may be at increased risk of acquiring MRSA compared to other infants. There are case reports of transmission of MRSA from parent to infant ${ }^{13-15}$; however, the risk is reduced with appropriate and frequent hand hygiene. A recent trial suggested that decolonizing parents may reduce the risk of parent-to-child $S$. aureus transmission, but further studies are needed to confirm this finding and to assess the impact on preventing infections in neonates. ${ }^{15,16}$ Empiric contact precautions are not necessary for infants with a parent known to have MRSA infection ${ }^{17,18}$ because no data suggest that this practice reduces MRSA transmission in the NICU. Specifically, any benefit of reducing transmission has not been shown to outweigh the potential unintended consequences of preemptive contact precautions. Units should strongly consider routine screening of infants (eg, weekly or biweekly) whose parent has a recent MRSA infection.

Measures to prevent MRSA transmission should not interfere with parental bonding. Healthcare personnel should encourage and promote skin-to-skin care and breastfeeding for MRSAcolonized and infected parents (see Question 1). The benefits of breastfeeding likely outweigh the risks of transmission of MRSA to noncolonized infants. If a parent has an active MRSA infection on the neck or chest (for skin-to-skin care); breast (for breastfeeding); or the hands (for all contact), the parent should ensure that the lesion is fully covered and should avoid any direct contact of the infant with the infected area until the lesion is healed. 
Contact precautions only apply to patients with MRSA. Standard precautions are indicated for MSSA. Finally, adherence to hand hygiene practices is the critical element needed to prevent MRSA transmission. Parental infection prevention education should be a routine part of the NICU plan of care.

Question 3: In cases of multiple gestations with discordant MRSA status (eg, one twin with MRSA colonization and the other twin without MRSA colonization), should healthcare personnel place discordant multiples on contact precautions, permit skin-to-skin care with parents, and/or institute breastfeeding restrictions?

\section{Answer 3:}

1. Contact isolation should be used for MRSA-colonized infants. ${ }^{19}$ Healthcare personnel should also consider use of contact precautions for the noncolonized infant(s) among discordant multiples.

2. Healthcare personnel should encourage skin-to-skin care to promote parent-infant bonding, while emphasizing hand hygiene to prevent organism transfer to the noncolonized infant.

3. Healthcare personnel may consider having parents perform skin-to-skin care with the noncolonized infant prior to performing skin-to-skin care with the MRSA-colonized infant.

4. Healthcare personnel should encourage breastfeeding regardless of an infant's MRSA status.

5. Units should strongly consider routine screening of noncolonized infants (eg, weekly, biweekly) with MRSA-colonized or infected siblings in the NICU.

Managing the care of multiple gestation infants with discordant MRSA status varies across institutions. There are case reports of transmission between siblings ${ }^{18,20}$; therefore, compared with other infants in the NICU, noncolonized infants who have MRSA-colonized siblings may be at increased risk of acquiring MRSA. Units should consider use of contact precautions empirically for the noncolonized neonate while acknowledging that the benefit of reducing transmission has not been shown to outweigh the potential unintended consequences of empiric contact precautions (eg, reduced interaction with healthcare personnel). Units should strongly consider routine screening of the noncolonized neonate to detect colonization (eg, weekly, biweekly). Management of MRSA-discordant infants should be based on the following principles: (1) contact precautions to prevent transmission should not interfere with parental bonding and skin-to-skin care; (2) the benefits of breastfeeding outweigh the low risk of MRSA transmission to noncolonized infants; (3) adherence to hand hygiene practices is critical to preventing $S$. aureus transmission; and (4) parental education about infection prevention should be incorporated into the routine plan of care. Parents should be informed about their possible role in the spread of MRSA between siblings and monitored to ensure hand hygiene compliance; however, healthcare personnel do not need to have parents clean their skin in between skin-to-skin care for infants with discordant MRSA status. Because standard precautions are indicated for MSSA, the preceding recommendations do not apply to infants colonized with MSSA.

Question 4: How long should healthcare personnel maintain contact precautions for MRSA-colonized neonates and what are reasonable criteria for discontinuation of contact precautions?

\section{Answer 4:}

1. Healthcare personnel should strongly consider continuing contact precautions for the duration of hospitalization in high-risk groups who are likely to remain MRSA-colonized, for example, infants with invasive (endotracheal tube) or noninvasive (nasal prongs) ventilation, a tracheostomy, or a draining/open wound.

a. Units should consider maintaining all MRSA-colonized infants on contact precautions for the duration of their hospitalization because of high rates of persistent and/or recurrent colonization.

2. Units may consider developing processes to document infants who have cleared MRSA through surveillance testing and to define criteria for removal of contact precautions.

3. Units should perform serial surveillance testing to document continued clearance of MRSA colonization if contact precautions are removed.

Some infants may remain MRSA-colonized for long periods, even infants that undergo treatment with topical antibiotics/ antiseptics (decolonization). Invasive devices, such as endotracheal or tracheostomy tubes, are reservoirs for MRSA and may promote persistent colonization. ${ }^{2,21,22}$ Because these infants may be ongoing reservoirs for MRSA transmission, units should consider continuing contact precautions for these infants while these devices remain in place. Other devices may serve as reservoirs for MRSA, such as gastrostomy tubes, but few data exist describing the natural history of colonization in infants with these devices. Infants with a history of MRSA-infected wounds may remain persistently colonized long after the wound has healed. However, certain infants' colonization status may change over time, such as after extubation or wound healing.

Infants with anticipated prolonged NICU stays can be periodically reassessed to determine whether they have persistent colonization or whether they can be cleared from contact precautions. A number of factors leads some units to consider removing infants from contact precautions: (1) infants' transient colonization; (2) potential negative consequences of contact precautions in reducing interaction with healthcare personnel, parent bonding, and increasing healthcare costs; and (3) the physical layout of the NICU (eg, private room vs. open bay). If units choose to remove infants with prior MRSA colonization from contact precautions, then the unit should define clear criteria for removal. Healthcare personnel should be aware that MRSA colonization can be intermittently missed using standard methods. As such, to document the absence of MRSA, collecting a minimum of 2 consecutive nares cultures at least a week apart increases the likelihood that the child is no longer colonized with MRSA. ${ }^{17,23}$ Units may consider screening infants who are not on antibiotics with activity against MRSA and do not have actively draining lesions or clinical symptoms of an MRSA infection for removal of contact precautions. When an infant with a known history of MRSA colonization or infection is readmitted to the NICU, clinicians should strongly consider using contact precautions and determine whether retesting for continued MRSA carriage is warranted. These recommendations do not apply to MSSA-colonized infants because MSSA-colonized infants are not routinely placed on contact precautions.

Question 5: Should healthcare personnel consider active surveillance cultures of hospitalized neonates for $S$. aureus and if so, what are the best strategies?

\section{Answer 5:}

1. No specific standard protocol exists for frequency of $S$. aureus surveillance testing, specific population(s) to test, or duration of active surveillance. 
2. For MRSA

a. Healthcare personnel should consider routine active surveillance when the results can inform infection prevention and control measures (eg, patient cohorting, contact precautions, and/or decolonization) and/or clinical management decisions (ie, antibiotic selection if subsequent invasive disease occurs).

b. In specific situations, healthcare personnel should perform active surveillance to inform additional infection prevention strategies when (1) an outbreak has been identified; (2) healthcare-associated transmission is ongoing and deemed of clinical importance; or (3) an individual patient may be at higher risk for colonization or infection (eg, prior to surgery, multiple gestation with differing MRSA colonization status, parent known to be a carrier or otherwise infected with MRSA, or outborn newborns from settings of higher prevalence).

3. For MSSA

a. Routine active surveillance has no clear benefit unless units use surveillance results to inform infection prevention and control measures (eg, decolonization).

b. In specific situations, healthcare personnel should consider performing active surveillance to inform additional infection prevention strategies (1) when an outbreak has been identified and (2) when there is a need to identify individual patients who may be at higher risk for infection (eg, low birth weight or prior to surgery).

4. Units should use culture-based or molecular methods for $S$. aureus surveillance, with anterior nares being the preferred site to sample. If multiple sites are sampled, units should consider performing a composite culture of all swabs.

5. Units should institute contact precautions for infants with positive MRSA test results. See Question 4 regarding duration of contact precautions.

6. Healthcare personnel should not routinely perform $S$. aureus testing of equipment and/or other environmental surfaces.

Nearly all studies that include data supporting the use of active surveillance cultures are observational in design. Typically, the studies include colonization as an outcome, and in some studies, active disease. ${ }^{24}$ Overall, although higher rates may be noted in outborn infants, MRSA colonization among NICU infants in the United States is between 0 and $4 \% .^{2}$ Although the anterior nares are the most frequent site for identification of $S$. aureus colonization, the addition of the umbilical, rectal, perirectal, or axillary sites may increase sensitivity. ${ }^{25}$ Patient samples can be cultured on blood agar and/or chromogenic selective agar for identification of S. aureus; however, molecular-based methods may improve sensitivity and rapid identification. ${ }^{26}$ Whether sampling additional sites and using more sensitive molecular-based methods is costeffective and reduces MRSA infections remains unknown.

In the outbreak setting, active surveillance for $S$. aureus is most useful when combined with other infection prevention and control measures such as contact precautions and targeted decolonization. ${ }^{24}$ In the outbreak setting, some facilities perform active surveillance on all new admissions, only outborn infants (MRSA only), on infants with bed spaces geographically close to infected babies, and/or all babies resident in the NICU at the time of surveillance. Active surveillance protocols vary. Repeated surveillance screening on a weekly or biweekly basis may be useful to confirm or exclude ongoing transmission, but if it is used as an isolated intervention, its effectiveness in preventing MRSA infections is uncertain.

In the nonoutbreak setting, asymptomatically colonized infants are a known reservoir for transmission and are still at risk for developing infection. ${ }^{2,17,24}$ Although data on patients in the NICU may be limited, studies report that placement of asymptomatic patients on contact precautions may have potential unintended consequences, such as reduced monitoring by staff. ${ }^{27}$ Units should balance the decision to perform active surveillance for MRSA with potential risks and associated costs. The costs and logistical requirements for routine $S$. aureus screening may be substantial, and the clinical value and cost-effectiveness of such approaches in the nonoutbreak setting are not well supported. Whether implementing other interventions, such as contact precautions in addition to performing active surveillance, prevents infections remains unclear. Limited data exist on the use of active MSSA surveillance or contact precautions to prevent infections in the NICU. ${ }^{28,29}$ Some prospective data show success with routine surveillance of new admissions followed by decolonization with nasal mupirocin to reduce invasive disease. ${ }^{30}$

Whether environmental contamination contributes to $S$. aureus acquisition in the NICU remains unclear. No data exist, in any age group, to suggest that environmental culturing provides useful information to prevent $S$. aureus transmission and infections. In the NICU setting, observational data suggest that those housed in a single room (vs. multibed pods) were less likely to acquire MRSA and MSSA. Thus, single- versus multiple-occupancy room is potentially a marker for environmental and/or staff contamination. ${ }^{31}$ In adult populations, MRSA colonization (not disease) may be more common in patients who reside in a room of a prior occupant known to have MRSA disease. ${ }^{32}$

Although healthcare personnel have been implicated as reservoirs propagating NICU MRSA outbreaks, routine healthcare personnel screening is not recommended unless epidemiologic data strongly suggest a possible link to transmission and other standard measures have been unsuccessful at controlling transmission.

Question 6: What are potential methods and indications for decolonizing NICU infants colonized with S. aureus?

\section{Answer 6:}

1. An optimal decolonization regimen for infants has not been determined. Intranasal mupirocin twice daily for 5-7 days is an acceptable method for $S$. aureus decolonization of infants.

2. Units should not use systemic antimicrobials as a decolonization strategy because this approach has not been studied in this population and it may select for antibiotic resistant organisms and may lead to adverse drug events.

3. Units may choose to perform targeted S. aureus decolonization in specific situations to reduce the risk of infection in colonized infants, for example, (1) when an outbreak has been identified and (2) when a colonized patient may be at higher risk for infection (eg, low birth weight, indwelling devices, or prior to high-risk surgeries).

4. The literature does not definitively determine the effectiveness of universal decolonization (treating all infants regardless of $S$. aureus colonization status) as a strategy to prevent $S$. aureus infections in NICUs.

Infants colonized with MRSA have an estimated $22 \%-28 \%$ risk of developing a MRSA infection.,21,32-34 Infants colonized with MSSA are also at increased risk of infection. ${ }^{30,31}$ In some 
populations, eradicating $S$. aureus colonization, via decolonization, can reduce the risk of infection. ${ }^{21,28,30,35}$ Both targeted and universal decolonization strategies have been employed in NICUs, but data are more limited for universal decolonization. Data showing efficacy and safety of $S$. aureus decolonization to reduce infection risk are primarily available from observational studies and a recent randomized trial that was not powered to detect a reduction in $S$. aureus infections. ${ }^{6,29,30,35-37}$

Even though decolonization has included treatment with topical antibiotics, topical antiseptics, and rarely, systemic antimicrobials, intranasal mupirocin alone is most commonly used. Intranasal mupirocin is not approved by the US Food and Drug Administration (FDA) for this indication in neonates, yet numerous studies reporting the use of mupirocin for $S$. aureus decolonization have not identified any clinically relevant adverse effects. ${ }^{28,30,38}$ Mupirocin can be applied twice daily for 5-7 days, although some centers report treating for longer. Although mupirocin intranasal application is extrapolated from studies in adult patients, no data exist on whether there is additional benefit to applying mupirocin to other body sites that may be colonized (eg, the umbilical stump, intertriginous areas, etc) among neonates. One study found a 5-day course of mupirocin applied to intranasal, periumbilical, and perianal areas to be safe and effective at reducing $S$. aureus colonization among NICU infants. ${ }^{30}$ Systemic antimicrobials have not been studied as part of decolonization regimens in infants.

Some units may choose to include topical antisepsis, most frequently with chlorhexidine gluconate (CHG). NICUs often use CHG for skin antisepsis; however, the FDA notes that these products should be "used with care in premature infants or infants under 2 months of age." 39 Despite widespread CHG use in NICUs, adverse events are infrequent. ${ }^{40-43}$ Concerns about skin irritation and the potential for systemic absorption have led to significant practice variations in CHG use; many NICUs restrict CHG application, especially in preterm infants within the first month of life. ${ }^{44}$

Healthcare personnel should consider decolonization in the following settings: outbreaks, periods with high rates of $S$. aureus transmission or infection despite other standard infection prevention measures, and the management of colonized infants at high risk of $S$. aureus infection such as preterm, low-birth-weight infants, infants with indwelling devices, and prior to high-risk surgery. When implementing a decolonization program, units must be thoughtful about possible unintended consequences including (1) changes in the microbial ecology in the intensive care unit (ICU), (2) alterations of the individual infant's microbiota, and (3) selection for antibiotic-resistant and antiseptic-tolerant strains. Commonly, S. aureus recolonization occurs following intranasal mupirocin. ${ }^{21,30,45}$ In settings for which decolonization is implemented, the risk of $S$. aureus disease among colonized infants should outweigh the potential consequences of decolonization noted above. Further studies are needed to determine the safety and efficacy of decolonization in infant populations, who will have the greatest benefit from decolonization, optimal decolonization regimens, and unintended consequences.

Question 7: Should NICUs use preemptive contact precautions for patients being screened for MRSA on admission?

\section{Answer 7:}

1. Units may choose not to use preemptive contact precautions for all NICU infants screened for MRSA at admission.

2. In outbreak settings or in NICUs with ongoing MRSA transmission, units may consider applying preemptive contact precautions for complex patients (eg, with central venous catheters, nonintact skin, or ventilator tubes) while admission $S$. aureus surveillance results are pending.

Preemptive contact precautions entail healthcare personnel wearing PPE while caring for the infant until surveillance culture results are finalized. If healthcare personnel perceive admitted neonates to be at high risk of MRSA colonization, then preemptive contact precautions may reduce the risk of exposure of healthcare personnel to MRSA. Small retrospective studies have suggested that undetected MRSA carriers that are not placed on contact precautions pose a significant risk to other patients within the unit. ${ }^{36}$ However, if healthcare personnel perceive admitted neonates to be at low risk of MRSA colonization, then preemptive contact precautions are likely not indicated. Some states mandate screening all neonates, even those at low risk of colonization. Therefore, units may choose not to use preemptive contact precautions for all infants admitted to their unit. The average onset of MRSA colonization among NICU infants is $21-30$ days of age; therefore, older infants who are admitted from another NICU or from the community may have a higher likelihood of MRSA colonization compared to newborn NICU admissions. ${ }^{2}$ Studies also suggest that the risk of multidrug-resistant organism colonization is 5-6 times higher after the first week of life. ${ }^{46}$ Therefore, preemptive contact precautions might be considered in older infants transferred after age 7 days. Still, in absolute terms, the risk is low and the costeffectiveness of screening older infants at admission is unknown. For this reason, the authors do not currently recommend routine preemptive contact precautions.

Similarly, use of preemptive contact precautions for infants colonized with MSSA has not been studied. Acquisition of MSSA parallels that of MRSA, with a median time to colonization in the third to fourth week of life. ${ }^{1}$ For this reason, the yield of surveillance cultures for MSSA in the first week of life is expected to be low. Studies evaluating the use of preemptive contact precautions in the context of optimized hand hygiene among healthcare personnel and routine active surveillance cultures showed little to no impact on transmission. ${ }^{47}$ One large observational study reported a significant reduction in endemic MRSA transmission with the use of preemptive contact precautions; however, there was also a marked increase in hand hygiene compliance rates during the study period that confounds the results. ${ }^{48}$ In outbreak settings, units should consider a multimodal approach to mitigating MRSA transmission within the NICU, focused primarily on optimal hand hygiene practices. This approach may also include preemptive contact precautions for select high-risk patients or older outborn infants.

Acknowledgments. The authors thank NICU Advisory Panel Chairs Drs. Kristina Bryant, Alexis Elward, and Deborah Yokoe, expert reviewers Karen Puopolo, MD, PhD, and Sandra Arnold, MD, as well as the expert reviewers from the American Academy of Pediatrics (AAP).

The authors would like to thank Valerie Deloney, MBA for her editorial assistance and organizational expertise in the development of this manuscript.

NICU Advisory Panel: Kenneth M. Zangwill, MD, American Academy of Pediatrics Section on Infectious Diseases (AAP/SOID); Nancy Foster, American Hospital Association (AHA); Katrina Crist, MBA, CAE, Association for Professionals in Infection Control and Epidemiology (APIC); Beth Huizinga, MSN, RNC-NIC, National Association of Neonatal Nurses (NANN); Pablo Sánchez, MD, Infectious Diseases Society of America (IDSA); Aaron M. Milstone, MD, Pediatric Infectious Diseases Society (PIDS); Margaret VanAmringe, MHS, The Joint Commission; Judith Guzman-Cottrill, DO, The Vermont Oxford Network (VON). 
Financial support. No financial support was provided relevant to this article.

Conflicts of interest. The following disclosures are a reflection of what has been reported to SHEA. To provide thorough transparency, SHEA requires full disclosure of all relationships, regardless of relevancy to the guideline topic. Evaluation of such relationships as potential conflicts of interest is determined by a review process. The assessment of disclosed relationships for possible conflicts of interest will be based on the relative weight of the financial relationship (ie, monetary amount) and the relevance of the relationship (ie, the degree to which an association might reasonably be interpreted by an independent observer as related to the topic or recommendation of consideration). The reader of this guidance should be mindful of this when the list of disclosures is reviewed.

K.M.Z. reports research funding from Harbor-UCLA National Institutes of Health Clinical and Translational Science Awards (NIH CTSA grant no. UL1TR0001881) and the Agency for Healthcare Research and Quality (AHRQ grant nos. 1R01HS025690-01 and R18CARB). J.B.C. reports research funding from the NIH CTSA (grant no. KL2 TR002646). A.M.M. reports consulting for Becton Dickinson and research funding from the NIH (grant nos. R21AI125179 and K24AI141580) and from the AHRQ (grant no. R01 HS022872) and from the CDC (grant no. U01CK000556). All authors report no conflicts of interest relevant to this article.

\section{References}

1. Shane AL, Hansen NI, Stoll BJ, Bell EF, Sánchez PJ, Shankaran S, et al. Methicillin-resistant and susceptible Staphylococcus aureus bacteremia and meningitis in preterm infants. Pediatrics 2012;129:e914-22.

2. Zervou FN, Zacharioudakis IM, Ziakas PD, Mylonakis E. MRSA colonization and risk of infection in the neonatal and pediatric ICU: a meta-analysis. Pediatrics 2014;133:e1015-e1023.

3. Ericson JE, Popoola VO, Smith PB, et al. Burden of invasive Staphylococcus aureus infections in hospitalized infants. JAMA Pediatr 2015;169:1105-1111.

4. Lake JG, Weiner LM, Milstone AM, Saiman L, Magill SS, See I. Pathogen distribution and antimicrobial resistance among pediatric healthcareassociated infections reported to the National Healthcare Safety Network, 2011-2014. Infect Control Hosp Epidemiol 2018;39:1-11.

5. Spaulding AB, Watson D, Dreyfus J, et al. Epidemiology of bloodstream infections in hospitalized children in the United States, 2009-2016. Clin Infect Dis 2018;69:995-1002.

6. Delaney HM, Wang E, Melish M. Comprehensive strategy including prophylactic mupirocin to reduce Staphylococcus aureus colonization and infection in high-risk neonates. J Perinatol 2013;33:313-318.

7. Achermann Y, Seidl K, Kuster SP, et al. Epidemiology of methicillinsusceptible Staphylococcus aureus in a neonatology ward. Infect Control Hosp Epidemiol 2015;36:1305-1312.

8. Madigan T, Cunningham SA, Patel R, et al. Whole-genome sequencing for methicillin-resistant Staphylococcus aureus (MRSA) outbreak investigation in a neonatal intensive care unit. Infect Control Hosp Epidemiol 2018;39: 1412-1418.

9. Johnson J, Quach C. Outbreaks in the neonatal ICU: a review of the literature. Curr Opin Infect Dis 2017;30:395-403.

10. Guyatt GH, Oxman AD, Vist GE, et al. GRADE: an emerging consensus on rating quality of evidence and strength of recommendations. BMJ 2008; 336:924-926.

11. Sandora TJ, Bryant KK, Cantey JB, Elward AM, Yokoe DS, Bartlett AH. SHEA neonatal intensive care unit (NICU) white paper series: practical approaches to Clostridioides difficile prevention. Infect Control Hosp Epidemiol 2018;39:1149-1153.

12. Munoz-Price LS, Banach DB, Bearman G, et al. Isolation precautions for visitors. Infect Control Hosp Epidemiol 2015;36:747-758.

13. Al-Tawfiq JA. Father-to-infant transmission of community-acquired methicillin-resistant Staphylococcus aureus in a neonatal intensive care unit. Infect Control Hosp Epidemiol 2006;27:636-637.

14. Pinter DM, Mandel J, Hulten KG, Minkoff H, Tosi MF. Maternal-infant perinatal transmission of methicillin-resistant and methicillin-sensitive Staphylococcus aureus. Am J Perinatol 2009;26:145-151.
15. Milstone AM, Voskertchian A, Koontz DW, et al. Effect of treating parents colonized with Staphylococcus aureus on transmission to neonates in the intensive care unit: a randomized clinical trial. JAMA 2019;323:319-328.

16. Zachariah P, Saiman L. Decreasing Staphylococcus aureus in the neonatal intensive care unit by decolonizing parents. JAMA 2019. doi: 10.1001/ jama.2019.20784.

17. Calfee DP, Salgado CD, Milstone AM, et al. Strategies to prevent methicillinresistant Staphylococcus aureus transmission and infection in acute care hospitals: 2014 update. Infect Control Hosp Epidemiol 2014;35 suppl 2: S108-S1032.

18. Geva A, Wright SB, Baldini LM, Smallcomb JA, Safran C, Gray JE. Spread of methicillin-resistant staphylococcus aureus in a large tertiary NICU: network analysis. Pediatrics 2011;128:e1173-e1180.

19. Siegel JD, Rhinehart E, Jackson M, Chiarello L, Committee HICPA. Management of multidrug-resistant organisms in health care settings, 2006. Am J Infect Control 2007;35:S165-S193.

20. Hollis RJ, Barr JL, Doebbeling BN, Pfaller MA, Wenzel RP. Familial carriage of methicillin-resistant Staphylococcus aureus and subsequent infection in a premature neonate. Clin Infect Dis 1995;21:328-332.

21. Popoola VO, Budd A, Wittig SM, et al. Methicillin-resistant Staphylococcus aureus transmission and infections in a neonatal intensive care unit despite active surveillance cultures and decolonization: challenges for infection prevention. Infect Control Hosp Epidemiol 2014;35:412-418.

22. Popoola VO, Tamma P, Reich NG, Perl TM, Milstone AM. Risk factors for persistent methicillin-resistant Staphylococcus aureus colonization in children with multiple intensive care unit admissions. Infect Control Hosp Epidemiol 2013;34:748-750.

23. Banach DB, Bearman G, Barnden M, et al. Duration of contact precautions for acute-care settings. Infect Control Hosp Epidemiol 2018;39:127-144.

24. Yokoe DS, Anderson DJ, Berenholtz SM, et al. A compendium of strategies to prevent healthcare-associated infections in acute care hospitals: 2014 updates. Infect Control Hosp Epidemiol 2014;35:967-977.

25. Rosenthal A, White D, Churilla S, Brodie S, Katz KC. Optimal surveillance culture sites for detection of methicillin-resistant Staphylococcus aureus in newborns. J Clin Microbiol 2006;44:4234-4236.

26. van Hal SJ, Jennings Z, Stark D, Marriott D, Harkness J. MRSA detection: comparison of two molecular methods (BD GeneOhm PCR assay and EasyPlex) with two selective MRSA agars (MRSA-ID and Oxoid MRSA) for nasal swabs. Eur J Clin Microbiol Infect Dis 2009;28:47-53.

27. Morgan DJ, Pineles L, Shardell M, et al. The effect of contact precautions on healthcare worker activity in acute care hospitals. Infect Control Hosp Epidemiol 2013;34:69-73.

28. Popoola VO, Colantuoni E, Suwantarat N, et al. Active surveillance cultures and decolonization to reduce Staphylococcus aureus infections in the neonatal intensive care unit. Infect Control Hosp Epidemiol 2016;37: 381-387.

29. Voskertchian A, Akinboyo IC, Colantuoni E, Johnson J, Milstone AM. Association of an active surveillance and decolonization program on incidence of clinical cultures growing Staphylococcus aureus in the neonatal intensive care unit. Infect Control Hosp Epidemiol 2018;39:882-884.

30. Kotloff KL, Shirley DT, Creech CB, et al. Mupirocin for Staphylococcus aureus decolonization of infants in neonatal intensive care units. Pediatrics 2019;143. doi: 10.1542/peds.2018-1565.

31. Washam MC, Ankrum A, Haberman BE, Staat MA, Haslam DB. Risk factors for Staphylococcus aureus acquisition in the neonatal intensive care unit: a matched case-case-control study. Infect Control Hosp Epidemiol 2018;39:46-52.

32. Huang SS, Datta R, Platt R. Risk of acquiring antibiotic-resistant bacteria from prior room occupants. Arch Intern Med 2006;166:1945-1951.

33. Maraqa NF, Aigbivbalu L, Masnita-Iusan C, et al. Prevalence of and risk factors for methicillin-resistant Staphylococcus aureus colonization and infection among infants at a level III neonatal intensive care unit. Am J Infect Control 2011;39:35-41.

34. Sakaki H, Nishioka M, Kanda K, Takahashi Y. An investigation of the risk factors for infection with methicillin-resistant Staphylococcus aureus among patients in a neonatal intensive care unit. Am J Infect Control 2009; $37: 580-586$ 
35. Wisgrill L, Zizka J, Unterasinger L, et al. Active surveillance cultures and targeted decolonization are associated with reduced methicillin-susceptible Staphylococcus aureus infections in VLBW infants. Neonatology 2017; 112:267-273.

36. Pierce R, Bryant K, Elward A, Lessler J, Milstone AM. Bacterial infections in neonates following mupirocin-based MRSA decolonization: a multicenter cohort study. Infect Control Hosp Epidemiol 2017;38:930-936.

37. Ristagno EH, Bryant KA, Boland LF, et al. Effect of intranasal mupirocin prophylaxis on methicillin-resistant Staphylococcus aureus transmission and invasive staphylococcal infections in a neonatal intensive care unit. Infect Control Hosp Epidemiol 2018;39:741-745.

38. Huang YC, Lien RI, Lin TY. Effect of mupirocin decolonization on subsequent methicillin-resistant Staphylococcus aureus infection in infants in neonatal intensive care units. Pediatr Infect Dis J 2015;34:241-245.

39. Safety and Effectiveness of Health Care Antiseptics; Topical Antimicrobial Drug Products for Over-the-Counter Human Use. Washington, DC: Food and Drug Administration; 2017: 0910-AH40.

40. Quach C, Milstone AM, Perpête C, Bonenfant M, Moore DL, Perreault T. Chlorhexidine bathing in a tertiary care neonatal intensive care unit: impact on central-line-associated bloodstream infections. Infect Control Hosp Epidemiol 2014;35:158-163.

41. Chapman AK, Aucott SW, Gilmore MM, Advani S, Clarke W, Milstone AM. Absorption and tolerability of aqueous chlorhexidine gluconate used for skin antisepsis prior to catheter insertion in preterm neonates. J Perinatol 2013;33:768-771.
42. Cleves D, Pino J, Patiño JA, Rosso F, Vélez JD, Pérez P. Effect of chlorhexidine baths on central-line-associated bloodstream infections in a neonatal intensive care unit in a developing country. J Hosp Infect 2018;100: e196-e199.

43. Neri I, Ravaioli GM, Faldella G, Capretti MG, Arcuri S, Patrizi A. Chlorhexidine-induced chemical burns in very low birth weight infants. J Pediatr 2017;191:262-265.e2.

44. Johnson J, Bracken R, Tamma PD, Aucott SW, Bearer C, Milstone AM. Trends in chlorhexidine use in US neonatal intensive care units: results from a follow-up national survey. Infect Control Hosp Epidemiol 2016;37: 1116-1118.

45. Akinboyo IC, Voskertchian A, Gorfu G, et al. Epidemiology and risk factors for recurrent Staphylococcus aureus colonization following active surveillance and decolonization in the NICU. Infect Control Hosp Epidemiol 2018;39:1334-1339.

46. Macnow T, O’Toole D, DeLaMora P, et al. Utility of surveillance cultures for antimicrobial resistant organisms in infants transferred to the neonatal intensive care unit. Pediatr Infect Dis J 2013;32:e443-e450.

47. Al Reyami E, Al Zoabi K, Rahmani A, Tamim M, Chedid F. Is isolation of outborn infants required at admission to the neonatal intensive care unit? Am J Infect Control 2009;37:335-7.

48. Morioka I, Yahata M, Shibata A, et al. Impact of pre-emptive contact precautions for outborn neonates on the incidence of healthcare-associated meticillin-resistant Staphylococcus aureus transmission in a Japanese neonatal intensive care unit. J Hosp Infect 2013;84:66-70. 